(2) Open Access Full Text Article

REVIEW

\title{
Stem cell technology for tendon regeneration: current status, challenges, and future research directions
}

This article was published in the following Dove Press journal:

Stem Cells and Cloning: Advances and Applications

II December 2015

Number of times this article has been viewed

\section{Pauline Po Yee Lui \\ Headquarter, Hospital Authority, Hong Kong SAR, People's Republic of China}

Correspondence: Pauline Po Yee Lui Headquarter, Hospital Authority, 9/F, Rumsey Street Carpark Building, 2 Rumsey Street, Sheung Wan, Hong Kong SAR, People's Republic of China Tel +85298094026

Email paulinelui00@gmail.com

\begin{abstract}
Tendon injuries are a common cause of physical disability. They present a clinical challenge to orthopedic surgeons because injured tendons respond poorly to current treatments without tissue regeneration and the time required for rehabilitation is long. New treatment options are required. Stem cell-based therapies offer great potential to promote tendon regeneration due to their high proliferative, synthetic, and immunomodulatory activities as well as their potential to differentiate to the target cell types and undergo genetic modification. In this review, I first recapped the challenges of tendon repair by reviewing the anatomy of tendon. Next, I discussed the advantages and limitations of using different types of stem cells compared to terminally differentiated cells for tendon tissue engineering. The safety and efficacy of application of stem cells and their modified counterparts for tendon tissue engineering were then summarized after a systematic literature search in PubMed. The challenges and future research directions to enhance, optimize, and standardize stem cell-based therapies for augmenting tendon repair were then discussed.
\end{abstract}

Keywords: stem cells, tendon repair, tendon tissue engineering, tendon injuries

\section{Introduction}

Tendon and ligament injuries are common clinical problems as a result of either overuse or aging. There are more than 30 million tendon and ligament injuries occurring annually worldwide. ${ }^{1}$ These injuries often upset the balance between mobility and stability of the joint which results in abnormal loading that could damage other soft tissues in and around the joint that can progress into early onset of osteoarthritis, pain, disability, and eventually the need for joint replacement. ${ }^{2}$ Their occurrence is particularly devastating to the elite athletes as it can be career-ending. The social and economic burden associated with these injuries presents a compelling argument to better understand their pathophysiology and develop appropriate treatments.

Tendon injury is currently managed by two approaches: 1) conservative treatment which aims to relieve pain and 2) surgical excision and repair. Irrespective of the approaches used, the treated tendon heals slowly and fails to regain its full function due to the formation of mechanically inferior scar tissue, ectopic bone, and adhesion or the lack of regeneration of fibrocartilage at the tendon to bone junction (TBJ). Repeated ruptures, joint stiffness, and restricted movement are common problems encountered even after repair.

The inability of tendon to self-repair and the inefficiency of current treatment regimens used clinically have sparked the exploration of alternative treatment strategies. The use of stem cells to repair tendon is particularly exciting and promising as stem cells 
have the potential to differentiate into tenocytes, show high proliferative and synthetic activities, and can secrete paracrine factors and exhibit immunomodulatory effects to promote tendon regeneration. However, a number of challenges have to be overcome before they can be used as a safe and effective therapeutic option for promoting tendon repair.

In this review, I aimed to present the recent advances, challenges, and future research directions of application of stem cells for tendon regeneration. I first recapped the anatomy of tendon. Then, I discussed the advantages and limitations of using different types of stem cells compared to terminally differentiated cells for tendon tissue engineering. Next, I summarized the literature regarding the safety and efficacy of application of stem cells and their modified counterparts for the promotion of tendon repair. Finally, I presented the challenges and future research directions to enhance, optimize, and standardize stem cell-based therapies for the augmentation of tendon repair.

\section{Why are tendons difficult to heal? A review of tendon anatomy}

Tendon consists of collagen (mostly type I collagen) and elastin embedded in a proteoglycan-rich matrix. Collagen and elastin account for $65 \%-80 \%$ and $1 \%-2 \%$, respectively, while proteoglycans account for $1 \%-5 \%$ of the tendon dry mass. ${ }^{3}$ The tendon matrix is produced by tenoblasts and tenocytes that lie parallel between the longitudinally-arranged collagen fibers. The cellularity of tendon tissue is low (as opposed to epithelial tissue which has high cellularity), explaining the low turnover and poor self-healing capacity of the tissue. Recent studies have shown that tendon also contains resident stem cells which function to maintain tendon homeostasis during growth and repair. ${ }^{4,5}$ Recent reports have also suggested that the change of tendon microenvironment after injury may induce erroneous differentiation of stem cells in tendon and cause pathological tendon ossification and failed tendon healing. ${ }^{6-8}$

The collagen molecules form cross-links and are packed in a quarter staggered fashion to form microfibrils, which are further aggregated together to form collagen fibrils. The staggering of collagen microfibrils and collagen fibrils produces the characteristic banding pattern of tendon under polarized microscopy. The collagen fibrils are grouped to form bigger units called collagen fibers. ${ }^{9}$ The endotenon, which is a sheath of connective tissue, interacts with each collagen fiber and binds the fibers together. The collagen fibers are further organized into higher orders of primary (subfascicle), secondary (fascicle), and tertiary fiber bundles to form the tendon. The entire tendon is surrounded by a thin connective tissue called epitenon. Some tendons (such as flexor tendon of fingers) are surrounded by a two-layer synovial sheath containing peritendinous fluid for lubrication as the tendons glide over the bone surfaces and prevention of bowstringing while some tendons (such as Achilles tendon) have the paratenon instead to reduce friction. The anatomy of tendon is illustrated in Figure 1.

Tendons contain blood vessels though it is considerably less than that in the other tissues such as muscles. The blood supply is particularly low in tendon regions that wrap around the bony pulleys. ${ }^{10}$ Such areas of diminished or absent blood supply are commonly the sites of tendon degeneration and/or rupture, suggesting that blood supply is important for tendon repair after injury. Blood vessels enter tendon at the myotendinous junction, which then supply the rest of the tendon. They run parallel to the fascicles as well as within the endotenon. It is still controversial if enthesis is an important site for the entry of blood vessels. ${ }^{11}$

The mid-substance of tendon is poorly innervated and the majority of nerve fibers are located within the tissue sheaths. ${ }^{12}$ However, nerve fibers can grow into damaged or ruptured tendons in association with blood vessels. The nerve fibers may function to regulate blood flow within the tendon, collect sensory information (including pain), and relay this to the central nervous system. ${ }^{12}$ The ingrowth of nerves into the injured tendon disappears as the tendon heals. ${ }^{13}$ The tendon enthesis is aneural and this may be associated with more frequent injuries at this region..$^{14}$

Due to the relatively acellular and avascular nature of tendon as well as the change of tendon microenvironment after injury, tendon repair is slow and often incomplete.

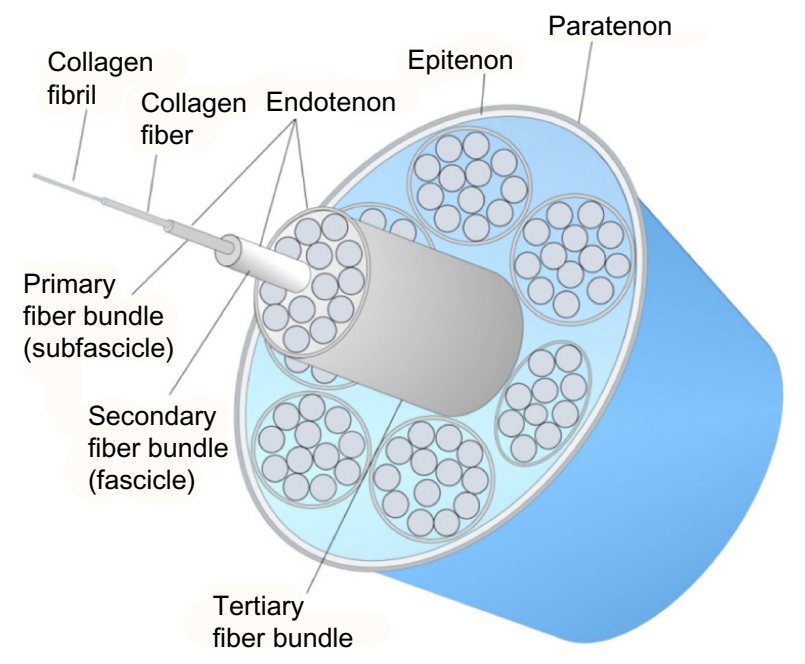

Figure I Anatomy of a tendon. 
Tendons naturally heal with the formation of scar tissue and ectopic bone which makes them prone to failure.

\section{Stem cell-based tendon tissue engineering}

Tissue engineering involves the use of cells, biomaterials, growth factors, enzymatic antagonists, or their combinations with the aim of directing a more sophisticated healing response to promote tissue repair. Both terminally differentiated cells and stem cells have been used in tendon tissue engineering. Among these different cell types, stem cells have attracted a great interest in tissue engineering as they can continuously reproduce themselves while maintaining the ability to differentiate into various cell types. The advantages and limitations of different cell types for tendon tissue engineering are discussed below.

\section{Advantages of stem cells compared to terminally differentiated cells for tendon tissue engineering}

As tenocytes are the major cell type in tendon and are the key machinery driving tissue repair via the production of growth factors and synthesis of extracellular matrix, it is logical to use tenocytes as the cell source in tendon tissue engineering. There are three primary problems associated with the use of tenocytes for tendon tissue engineering. First, tenocytes are highly differentiated cells and have limited capacity to replicate and differentiate. The intrinsic healing ability of tendon fibroblasts is poor. Zhang and Wang have reported that tendon-derived stem cells (TDSCs) proliferated faster compared to tenocytes. ${ }^{15}$ Second, phenotype drift and function loss are often observed during in vitro expansion of tenocytes. ${ }^{16,17}$ The tenocytes became more rounded, the confluent cell density decreased, the ratio of type III to type I collagen increased while the expression of decorin decreased during in vitro cell passaging. ${ }^{16}$ Tendon-selective genes including tenomodulin and thrombospondin 4 were rapidly downregulated in primary tendon fibroblasts cultured in monolayers and in organ cultures. ${ }^{18}$ Third, tendons are relatively acellular and contain few tenocytes and there is the problem of donor site morbidity in autologous transplantation. As tenocytes are fibroblasts, some research groups use skin fibroblasts which are more easily accessible for tendon repair. However, skin fibroblasts are not specialized cells for maintaining the homeostasis of tendon. The first two problems associated with the use of differentiated cells remain. Both cell sources have a low risk of teratoma formation and hence have fewer safety concerns. There have been clinical trials reporting the use of tenocytes ${ }^{19}$ and skin fibroblasts ${ }^{20}$ for the treatment of tendinopathy with promising results. Ultrasound-guided autologous tenocyte injection was reported to improve the clinical function and magnetic resonance imaging (MRI) tendinopathy scores in patients with refractory lateral epicondylitis at 4.5 years posttreatment. ${ }^{19}$ On the other hand, ultrasound-guided injection of autologous skin fibroblasts was reported to improve the Victorian Institute of Sport Assessment score in patients with patellar tendinopathy at 6 months posttreatment. ${ }^{20}$

Embryonic stem cells (ESCs) have unlimited proliferative capacity and theoretically can be induced into all types of somatic cells for tissue regeneration. However, there is a risk of teratoma formation after transplantation. ${ }^{21}$ Therefore, predifferentiation of ESCs to mesenchymal lineages is required prior to transplantation. As ESC-based therapies are particularly susceptible to generating tumors if the graft contains any undifferentiated cells, strategy to effectively remove the undifferentiated cells is needed. ${ }^{22}$ There is also an ethical concern with the harvest of ESCs from embryos for tendon repair. The generation of induced pluripotent stem cells (iPSCs) from terminally differentiated cells eases the ethical concern of using ESCs. However, the risk of teratoma formation remains as iPSCs are pluripotent. Moreover, reprogramming factors associated with cell proliferation and tumorigenesis as well as integrated viral vectors are often used for the generation of iPSCs, ${ }^{23,24}$ although there are now better and more efficient nonintegrating viral and nonviral vectors as well as small molecules for iPSC generation. ${ }^{24,25}$ Similar to ESCs, predifferentiation of iPSCs to cells of mesenchymal lineages followed by separation of the differentiated cells from the residual pluripotent progeny are required prior to transplantation. ${ }^{23}$ It is generally agreed that the differentiated cells derived from syngeneic iPSCs are not immunogenic and are not rejected after transplantation ${ }^{26,27}$ although one earlier study has reported that undifferentiated iPSCs could induce immune response in the syngeneic recipient mice after transplantation. ${ }^{28}$ Transplantation of ESC- or iPSC-derived tissues from an unrelated (allogeneic) donor that expresses foreign human leucocyte antigens may cause immunological rejection. Hence, the creation of stem cell banks comprising human leucocyte antigen-typed human ESCs and iPSCs is proposed to overcome the immunological barrier by providing human leucocyte antigen-matched (histocompatible) tissues for the target population. ${ }^{29}$

Unlike ESCs and iPSCs, mesenchymal stem cells (MSCs) have restricted self-renewal and lineage differentiation potential and hence have fewer safety concerns. The harvest of 
MSCs is easy and does not raise an ethical issue when compared to ESCs. MSCs have higher proliferative and synthetic activities compared to terminally differentiated cells. For instances, bone marrow-derived stem cells (BMSCs) were reported to show higher collagen production and cell proliferation (as indicated by higher DNA content) after seeding on polylactide/glycolide suture material compared to anterior cruciate ligament and skin fibroblasts. ${ }^{30}$ Similarly, others have reported that BMSCs possessed higher proliferation rate and collagen excretion in vitro as well as longer survival in the knee joint after transplantation when compared to anterior cruciate ligament or medial cruciate ligament fibroblasts. ${ }^{31}$ BMSCs, but not tendon fibroblasts, were reported to stimulate biological and biomechanical healing of patellar tendon after injury. ${ }^{32}$ MSCs are immune-privileged cells and hence allogeneic cells can be used for tendon repair, ${ }^{33-35}$ although they also retain a degree of immunogenicity in some circumstances that may limit their longevity and attenuate their beneficial effects. ${ }^{36}$ Recent studies have shown that MSCs had immunosuppressive effects. They were reported to switch the macrophage phenotype from pro-inflammatory to anti-inflammatory and secrete bioactive molecules that might promote tissue repair and disease treatment. ${ }^{37-40} \mathrm{MSCs}$ have been isolated from almost all types of tissues in the body. However, studies have shown variations in the stem cell properties of MSCs derived from different tissues. ${ }^{41}$ Some MSC types therefore may perform better compared to the other types for tendon repair. Despite the encouraging findings of MSC transplantation for tendon repair, the transplantation of BMSCs into the rabbit tendon defect was reported to form ectopic bone in some tendon samples. ${ }^{42,43}$ The transplanted BMSCs were found in the ectopic bone and expressed alkaline phosphatase, suggesting that some osteoblasts within the ectopic bone were derived from the implanted BMSCs. ${ }^{42,43}$ While the authors have reported that the seeding density tested $(1,4$, and 8 million cells $/ \mathrm{mL})$ did not affect the extent and frequency of ectopic bone formation after transplantation, they have speculated that the cell to collagen ratio and in vitro construct contraction might influence cell-cell contact and hence chances of ectopic bone formation after transplantation. ${ }^{43}$ Tumor induction in mice after transplantation of undifferentiated BMSCs was reported when the cells were transplanted in scaffolds to syngeneic and immune-deficient recipients. ${ }^{44} \mathrm{~A}$ recent study has shown that ESCs survived in greater numbers (constant number up to day 90 vs less than 5\% at day 10) and migrated to longer distance compared to BMSCs after injection into damaged horse tendons. ${ }^{45}$ Whether this difference in survival rate and distribution affected the healing outcomes has not been reported. Further study is needed. Table 1 summarizes the advantages and limitations of different cell sources for tendon tissue engineering.

\section{Stem cells as vehicles for gene therapy and sustained delivery of bioactive factors}

Although the clinical outcomes after stem cell transplantation were often encouraging, the mode of action of the transplanted cells remains unresolved. While it is generally assumed that stem cells promote tissue repair by direct engraftment and differentiation, differentiation of stem cells into target cell types is rarely shown. Furthermore, some studies have reported that only a small proportion of the cells persisted at the target sites and most of the cells were not detectable after 7-14 days posttransplantation. ${ }^{34,35}$ There are evidences to suggest that stem cells may promote tissue repair via the secretion of paracrine factors that reduce inflammation and promote angiogenesis. ${ }^{34,35,37}$ Microvesicles $(100-1,000 \mathrm{~nm})$ are membranous structures that arise from the budding of the plasma membrane ${ }^{46} \mathrm{~A}$ wide range of molecules including cytokines, growth factors as well as miRNA were identified in microvesicles derived from MSCs. Indeed, microvesicles were shown to promote the regeneration of a number of tissues (eg, lung, kidney, heart, liver, or nervous tissues) via transferring cytokines to the target or neighboring cells..$^{47,48}$ The production of microvesicles by stem cells may partially explain the paracrine effects observed in stem cell-based therapies. The use of cells that are either naturally or genetically engineered to provide a sustained delivery of multiple growth factors for tissue repair has an advantage over the application of single growth factor, which typically only has a half-life of minutes to hours. Stem cells therefore are useful as carriers of drugs and bioactive factors. ${ }^{49}$

\section{Current status of application of stem cells for tendon repair}

The PubMed database was searched with the key words "stem cells tendon" on July 26-29, 2015 and "bone marrow stromal cells tendon" on August 17, 2015 with no restriction in language and year of publication. The studies were selected after reviewing the titles and abstracts. Original studies evaluating the safety and efficacy of stem cell for the promotion of TBJ repair of rotator cuff and Achilles tendon as well as tendon repair in animals and human were included. A total of 857 and 351 articles were identified with the key words, respectively. Of these, 112 studies are eligible for inclusion in this review. Supplementary material 


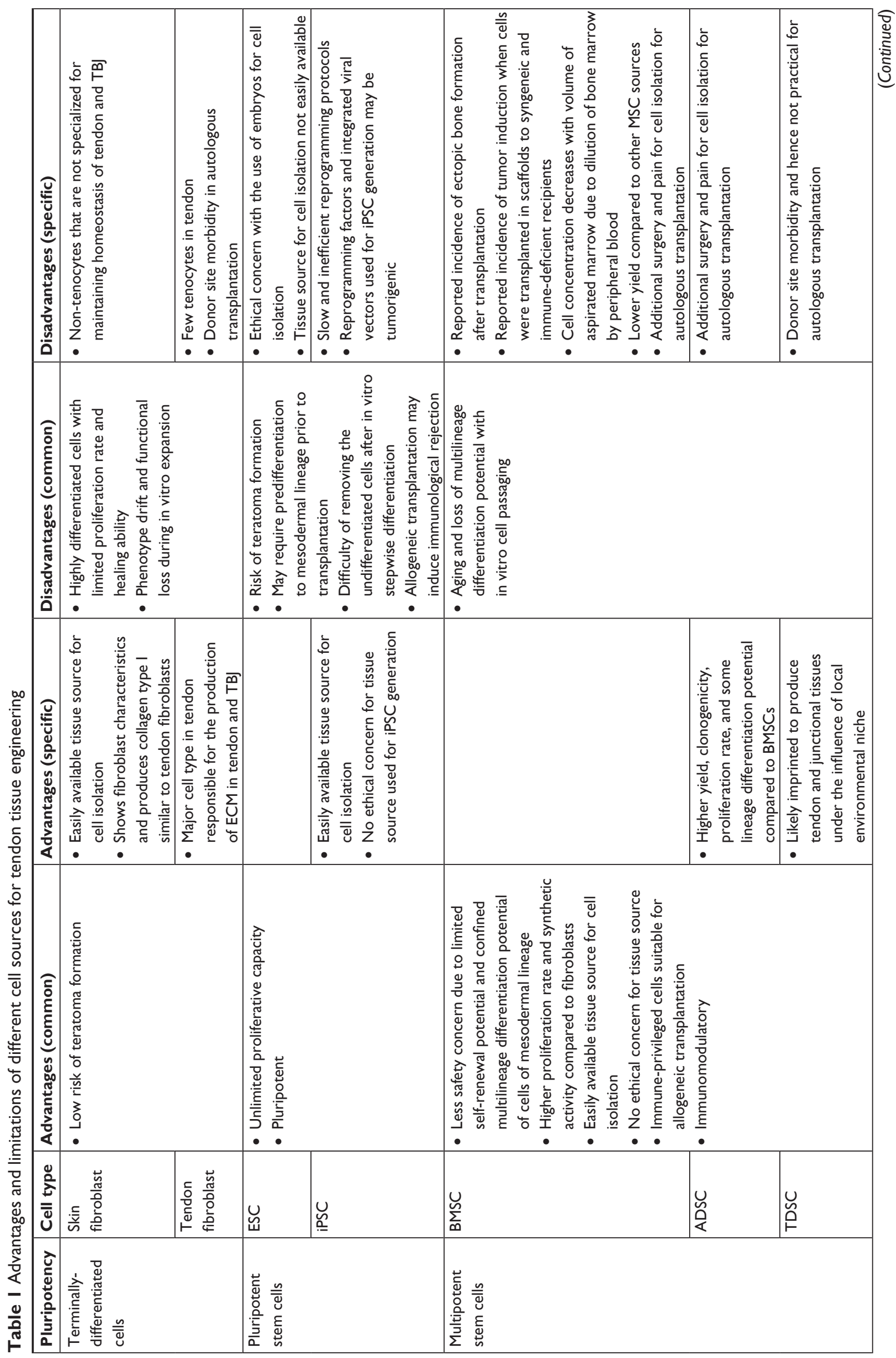




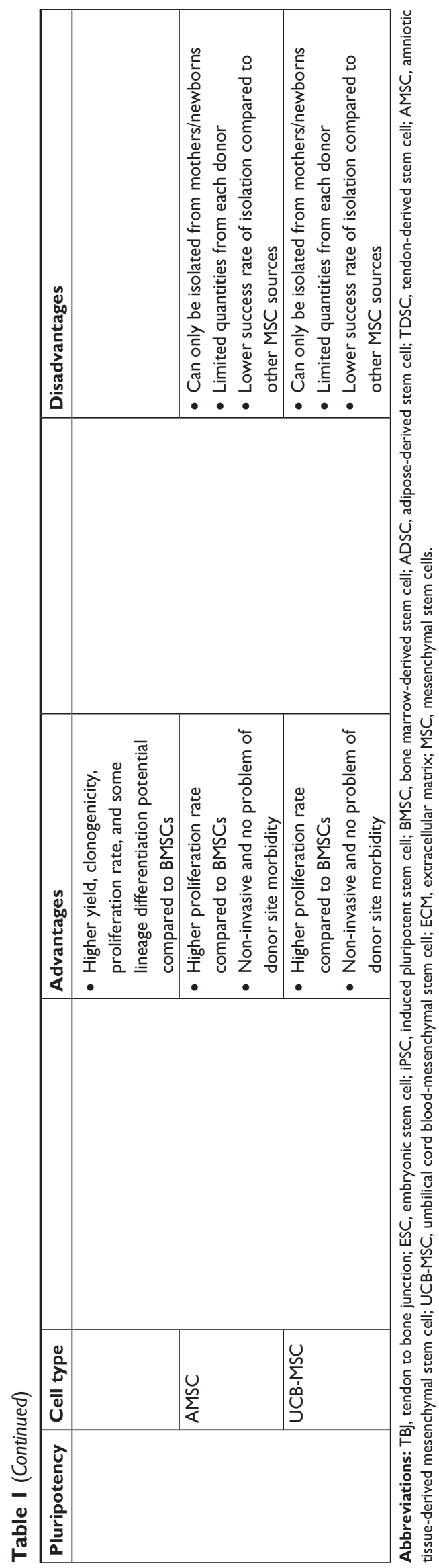

summarizes the preclinical and clinical studies (rows in gray color) on the application of stem cells for the promotion of tendon repair.

BMSCs, blood-derived MSCs, adipose-derived stem cells (ADSCs), TDSCs, ESCs, umbilical cord blood-MSCs, amniotic MSCs, ESCs, and iPSCs were the commonly used stem cell types for tendon repair. Ninety-nine studies are animal studies and five are clinical trials (Supplementary material). Achilles tendon transection or segmental tendon defects, patellar tendon window injury, collagenase-induced Achilles or superficial digital flexor tendon injury, naturally-occurring superficial digital flexor tendon injury and TBJ injury at the Achilles, infraspinatus or supraspinatus tendons were the commonly used tendon injury models. Most of the animal studies were based on small animal models such as rats and rabbits. The large animal models were collagenase-induced or naturally-occurring tendon injury in sheep or horses and most of them were on horses. Injury of the superficial digital flexor tendon is very common in horses and this explains why it is often used as a model of tendinopathy. Instead of injecting or transplanting stem cells, one study injected the conditioned medium of amniotic membrane-derived mesenchymal progenitor cells to promote healing of naturally-occurring tendon injuries in horses and reported a significant lower rate of reinjury compared to the untreated animals..$^{40}$ Except ten studies, the other preclinical studies reported the benefits of transplanting stem cells on tendon repair (Supplementary material). All the large animal studies have shown some positive effects of stem cell transplantation on tendon repair although there were variations in the quality, outcome assessments, and follow-up duration of the studies. While Gulotta et al, ${ }^{50}$ Valencia Mora et $\mathrm{al}^{51}{ }^{51}$ and Barco et $\mathrm{a}^{52}$ have reported that the transplantation of BMSCs did not improve supraspinatus TBJ healing in rat models, transosseous drilling of the greater tuberosity to release BMSCs to the suture zone was shown to improve supraspinatus TBJ repair. ${ }^{53}$ The transplantation of BMSCs was reported to improve Achilles $\mathrm{TBJ}^{54}$ and infraspinatus $\mathrm{TBJ}^{55}$ repair whereas the transplantation of ADSCs was reported to improve subscapularis TBJ repair. ${ }^{56}$ The follow-up duration of the animal studies was usually less than 12 weeks and hence short (Supplementary material).

Five clinical trials reported the use of stem cells for the promotion of tendon repair (chronic tendinopathy, three; rotator cuff tear, two) with promising results (Supplementary material). There are two clinical studies reporting the safety of using allogeneic stem cells for the promotion of tendon repair. The injection of allogeneic ADSCs for the treatment of chronic lateral epicondylosis was reported to 
be safe and effectively improved elbow pain, performance, and structural defects after 1 year in a small uncontrolled trial of 12 patients. ${ }^{57}$ The ultrasound-guided injection of allogeneic human placenta-derived mesenchymal stromal cells was also reported to be safe in six patients with refractory Achilles tendinopathy at 4 weeks after administration. ${ }^{58}$ Except the study by Hernigou et al, ${ }^{59}$ the sample sizes in the other clinical studies were small and there were no control groups (Supplementary material).

Since ectopic bone was formed in some tendon samples after transplantation of $\mathrm{BMSCs},{ }^{42,43}$ it is hypothesized that driving the MSCs toward the tenogenic lineage prior to transplantation may reduce the chance of ectopic bone and tumor formation as well as promote better tendon repair. Pretreatment of MSCs and MSCs derived from ESCs with growth factors (such as bone morphogenetic protein-12, connective tissue growth factor, platelet-rich plasma), ${ }^{60,61}$ overexpression of key transcription factors (such as smad8, scleraxis $[\mathrm{Scx}]$, early growth response protein 1, mohawk [Mkx], membrane type 1-matrix metalloproteinase 1), ${ }^{6-68}$ mechanical stimulation, ${ }^{69-71}$ topographical cues of scaffolds (such as acellular tendon matrix, aligned chitosan-based ultrafine fibers), ${ }^{72,73}$ or their combinations ${ }^{74}$ have been used to promote tenogenic differentiation of stem cells prior to delivery. The current evidences suggested that most of these attempts were successful with better healing compared to the transplantation of untreated stem cells.

Pretreatment of BMSCs with bone morphogenetic protein-12 was reported to promote their tenogenic differentiation and efficacy of tendon repair after transplantation. ${ }^{60}$ Platelet-rich plasma contains many growth factors and was reported to promote tenogenic differentiation of TDSCs. ${ }^{75}$ Except one study, ${ }^{76}$ combined treatment with stem cell and platelet-rich plasma was reported to enhance tendon repair compared to either one alone. ${ }^{61,77-80}$

Overexpression of key transcription factors of tendon development such as smad8, Scx, early growth response protein 1, and Mkx in MSCs was reported to promote their tenogenic differentiation and accelerate tendon and TBJ repair after transplantation (Supplementary material). In this regard, overexpression of a biologically active smad8 was reported to promote the tenogenic differentiation of a mouse MSC line that coexpressed the osteogenic gene (BMP2). ${ }^{62}$ The transplantation of the resulting engineered cells to the injury site was reported to promote better healing in two rat Achilles tendon defect models. ${ }^{62,63}$ The transplantation of MSCs overexpressing early growth response protein 1 was reported to promote junctiona ${ }^{64}$ and mid-substance ${ }^{65}$ tendon repair. The transplantation of MSC sheets overexpressing Mkx also promoted Achilles tendon repair in a mouse model. ${ }^{66}$ While BMSCs overexpressing bone morphogenetic protein-13 did not improve supraspinatus TBJ healing, ${ }^{81}$ BMSCs overexpressing $\mathrm{Scx}^{67}$ or membrane type 1-matrix metalloproteinase 1 (a developmental protein) ${ }^{68}$ were reported to improve healing in the same model.

Tendon is a mechanosensitive tissue. Appropriate tensile loading is required to maintain its composition and biomechanical properties. Tensile loading therefore has been used to promote the tenogenic differentiation and biomechanical properties of the tissue engineered constructs containing stem cells. Mechanical loading of BMSCs ${ }^{69,70}$ and TDSCs ${ }^{71}$ seeded in scaffolds prior to transplantation was reported to promote better tendon repair compared to the transplantation of the same unloaded cell constructs.

Biomaterial provides biological and architectural cues that can direct important cell behaviors and resulting tissue formation. ${ }^{82}$ Different types and designs of scaffolds have been used as vehicles and/or as niche signal to drive tenogenic differentiation of stem cells with promising results in vitro and in animal studies. In this regard, acellular tendon matrix components, nanosized fibers, and aligned fibers have been shown in many studies to promote tenogenic differentiation of the seeded stem cells and the resulting constructs were reported to promote better tendon repair. ${ }^{72,73}$

Stem cells have also been used as carriers for growth factors to promote tendon repair (Supplementary material). The transplantation of BMSCs transfected with transforming growth factor- $\beta 1$, but not vascular endothelial growth factor, promoted tendon repair. ${ }^{83}$ Another study has reported that the transplantation of BMSCs had negative effects and overexpression of basic fibroblast growth factor in BMSCs had negligible effects on Achilles tendon healing. ${ }^{84}$

\section{Challenges and future research directions to enhance/optimize/ standardize stem cell-based therapies to promote tendon repair Optimal stem cell source}

Further studies are needed to compare if the transplantation of stem cells isolated from different tissues exhibited difference in outcomes of tendon healing in animal models. As ESC-based therapies are particularly susceptible to generating tumors if the graft contains any undifferentiated cells, strategy to effectively remove the undifferentiated cells is needed. 


\section{Large animal models and clinical trials with long follow-up time}

While the application of stem cells for the promotion of tendon healing is promising in small animal models, there have been few well-controlled large animal studies and clinical trials (Supplementary material). The follow-up duration in animal studies was usually short (usually 4-12 weeks). Further research on the efficacy and safety of stem cell-based therapy for tendon repair in well-designed large animal models with extended follow-up time and randomized controlled clinical trials is needed.

\section{Stem cell delivery methods}

Studies comparing the use of different scaffolds for stem cell delivery in tendon repair are lacking. Further studies are needed to select the optimal scaffold for tendon repair. Delivery of stem cells via circulation is relevant for injury sites that are difficult to access such as the brain and the heart. Intralesional injection or transplantation is currently the commonly used administrative route for stem cell-based tendon repair as the tendons that are commonly injured (such as patellar tendon, Achilles tendon, and rotator cuff tendon) are located superficially. The administrative route may affect the distribution of available cells at the injury sites and clinical outcomes. The current limited evidence did not support the delivery of stem cells to the injured tendons via the circulatory system and regional perfusion. ${ }^{85,86}$ Sole et al have reported that the MSC number in lesion site was lower when the cells were delivered via the circulatory system compared to the intralesional injection in an equine tendon injury model. ${ }^{85}$ Moreover, arterial thrombosis was reported in the intravenously and intra-arterial perfused limbs ${ }^{85}$ Becerra et al have traced and compared the distribution of labeled BMSCs, that were delivered intralesionally, intravenously, or by regional perfusion, to the lesion site in an equine naturally-occurring tendon or ligament injury model ${ }^{86}$ Their results have shown that intralesional transplantation retained the highest number of BMSCs. ${ }^{86}$ BMSCs were largely distributed to the lung fields and there were no detectable cells in the tendon lesions after intravenous injection. ${ }^{86}$ This suggested that the cells could not "home" to the damaged tendon after intravenous administration. BMSCs could be detected in the lesion sites following regional perfusion though at a lower level compared to the intralesional injection. The cell labeling efficiency in the previous study was reported to be low and vary greatly (range from $1.3 \%$ to $18.5 \%$, mean $7.2 \%$ ). ${ }^{86}$ The follow-up duration was also short (24-48 hours). ${ }^{85,86}$ Further studies are needed to confirm the research findings of the previous studies as well as to understand the safety and healing outcomes (eg, ectopic bone formation) of different delivery routes.

\section{Optimal cell density}

There have been limited studies on the effects of cell density on tendon repair. One study has shown that collagen gel constructs seeded with higher density of BMSCs contracted faster and the seeded cells were better aligned after 3 days in vitro. ${ }^{87}$ Awad et al have compared the healing effect of transplanting 1, 4, and 8 million cells $/ \mathrm{mL}$ of autologous BMSCs seeded in collagen gel at the same cell to collagen ratio in a patellar tendon injury model.$^{43}$ They have reported that while the transplantation of BMSCs promoted tendon healing compared to natural repair, increasing the cellseeding density did not produce additional histological and biomechanical benefits. ${ }^{43}$ The authors have hypothesized that stress shielding produced by the tendon struts adjacent to the repair site might have reduced any benefits that would have accrued from increasing the seeding density of the implants in vivo. ${ }^{43}$ The current limited data show that the initial seeding density is independent of ectopic bone formation as ectopic bone was formed in $28 \%$ of BMSC-treated tendons, regardless of cell concentration $(1,4$, and 8 million cells $/ \mathrm{mL}){ }^{43}$ Nevertheless, the cell to collagen ratio and in vitro construct contraction may contribute to ectopic bone formation. Further studies in different tendon injury models are needed to identify the cell density that is most efficacious yet safe for tendon repair.

\section{Study on the fate and healing mechanisms of stem cells}

The fate of stem cells after transplantation remains unclear. Some studies have reported the disappearance of the transplanted cell $\mathrm{s}^{35,88}$ while other studies have reported detecting the transplanted cells within the tendon injury site in the animal models but the follow-up time after transplantation was usually short. ${ }^{89,90}$ MSCs might promote scarless tendon and TBJ repair via the secretion of a variety of bioactive factors that suppress inflammation, inhibit fibrosis and apoptosis, enhance angiogenesis, and stimulate mitosis and differentiation of host reparative cells..$^{35,37,40,91}$ The transplantation of MSCs was reported to reduce the infiltration of inflammatory cells and promote tendon and TBJ repair in animal models. ${ }^{35,91}$ The immunomodulatory role of MSCs on scarless tendon repair has been best demonstrated by the promotion of tendon and ligament repair with the injection of conditioned medium of amniotic membrane-derived mesenchymal 
progenitor cells in horses ${ }^{40}$ and the switching of macrophages from a pro-inflammatory to an anti-inflammatory phenotype after the addition of ADSCs in the macrophage and tendon fibroblast coculture. ${ }^{37}$ Better understanding of the fate and healing mechanisms, including immunomodulatory roles, of stem cells after transplantation to the tendon injury site would facilitate treatment monitoring and enhancement.

Fetal tendon heals with scarless tissue formation. Many key transcription factors (such as Scx and Mkx) and growth factors (such as transforming growth factor- $\beta 2$ ) for embryonic tendon development are also expressed in adult tendon. ${ }^{92}$ Better understanding of the process of embryonic tendon development therefore can help the identification of novel therapeutic strategies, including stem cell-based therapy, to recapitulate critical aspects of tenogenesis and effectively direct the cells to differentiate and regenerate scarless tissues in adult tendon after injury. ${ }^{93,94}$ Recently, the idea of mobilizing endogenous stem cells for regenerating tendon tissues has emerged. ${ }^{95,96}$ Further understanding of the role of stem cells in natural tendon healing is warranted to explore strategies to promote tendon regeneration without cell transplantation.

\section{Manufacturing and regulatory considerations}

The fact that the product of stem cell-based therapy is the living cells, not their isolated and enriched protein products, brings forward a different set of manufacturing and regulatory challenges. Specific issues associated with cell transplantation include scaling-up of production, biological or donor-todonor variability, immunological responses to alloantigens, and tumorigenicity of the transplanted cells.

The number of stem cells required for tissue repair in the clinical setting is often greater than $10^{6}-10^{7}$ cells. ${ }^{57,58}$ Safe, robust, and cost-effective strategies for scaling up the production of stem cells are required for translating the stem cell-based strategies from animal models to clinical trials. Optimization of cell culture system is needed for increasing the product quantity (ie, cell number) while maintaining its quality (eg, cell fate). Oxygen plays an important role in stem cell proliferation, maintenance, and differentiation..$^{97,98}$ Other parameters, such as $\mathrm{pH}$, osmolarity, medium shear, and medium composition also have important effects on stem cell quantity and quality. These parameters need to be optimized and standardized to increase the stem cell yield while ensuring its safety.

Robust assays capable of product characterization, process validation and control, and predicting clinical safety and efficacy are required for clinical application of stem cells. Identification of surrogate markers of cellular function and/or culture performance is required. This is not trivial. While the expression of transcription factors Oct4, nanog, and sox 2 are relatively reliable pluripotency markers (albeit intracellular) of ESCs and iPSCs, reliable markers of adult stem cells are rare. This is partly due to the poor understanding of the stemness functions of the current adult stem cell markers. Further studies on the stemness functions of markers commonly used for defining adult stem cells are needed. Although many MSC types were reported to exhibit low immunogenicity, ${ }^{33-35}$ emerging studies also have reported that MSCs could adopt an immunogenic phenotype and stimulated immune cells. ${ }^{99}$ Further research on the immunogenicity of MSCs is needed.

\section{Conclusion}

Due to its relatively low cellularity and vascularity as well as the change in the tissue microenvironment after injury, tendons form scar tissue and ectopic bone after injury without regenerating the original tendon structure. Tissue engineering with stem cells offers the potential to replace the injured/damaged tissues with healthy and functional ones. The use of stem cells for tendon tissue engineering is advantageous compared to terminally differentiated cells as stem cells are pluripotent or multipotent, highly proliferative and synthetic, and can provide the appropriate signals to promote tendon regeneration compared to terminally differentiated cells. Moreover, stem cells can also be used as a vehicle for gene therapy and sustained delivery of bioactive factors for tendon repair. The previous animal studies have shown that stem cells and their modified counterparts were generally safe and effective for tendon repair, with the exception of the presence of ectopic bone and tumor in some studies. Many challenges have to be overcome before stem cells can be used as a safe and effective therapeutic option to promote tendon repair. Well-controlled large animal models with extended follow-up period and randomized controlled clinical trials are needed to evaluate the longterm safety and efficacy of stem cell-based products. The optimal scaffold, stem cell type, method of cell delivery, and cell density for tendon repair need further research. Translating stem cell-based therapies from bench to bed requires overcoming significant cell-manufacturing and regulatory challenges. Better understanding of the healing mechanisms of stem cell-based therapies and factors of embryonic tendon development would provide cues to promote tendon regeneration. 


\section{Disclosure}

The author reports no conflicts of interest in this work.

\section{References}

1. Maffulli N, Wong J, Almekinders LC. Types and epidemiology of tendinopathy. Clin Sports Med. 2003;22(4):675-692.

2. Jung HJ, Fisher MB, Woo SL. Role of biomechanics in the understanding of normal, injured, and healing ligaments and tendons. Sports Med Arthrosc Rehabil Ther Technol. 2009;1(1):9.

3. Hess GP, Cappiello WL, Poole RM, Hunter SC. Prevention and treatment of overuse tendon injuries. Sports Med. 1989;8:371-384.

4. Bi Y, Ehirchiou D, Kilts TM, et al. Identification of tendon stem/progenitor cells and the role of the extracellular matrix in their niche. Nat Med. 2007;13(10):1219-1227.

5. Tan Q, Lui PP, Lee YW. In vivo identity of tendon stem cells and the roles of stem cells in tendon healing. Stem Cells Dev. 2013;22(23):3128-3140.

6. Lui PP. Histopathological changes in tendinopathy - potential roles of BMPs? Rheumatology (Oxford). 2013;52(12):2116-2126.

7. Lui PP, Chan KM. Tendon-derived stem cells (TDSCs): from basic science to potential roles in tendon pathology and tissue engineering applications. Stem Cell Rev. 2011;7(4):883-897.

8. Rui YF, Lui PP, Chan LS, Chan KM, Fu SC, Li G. Does erroneous differentiation of tendon-derived stem cells contribute to the pathogenesis of calcifying tendinopathy? Chin Med J (Engl). 2011;124(4):606-610.

9. Jozsa L, Balint BJ, Reffy A, Demel Z. Histochemical and ultrastructural study of adult human tendon. Acta Histochem. 1979;65:250-257.

10. Petersen W, Hohmann G, Stein V, Tillmann B. The blood supply of the posterior tibial tendon. J Bone Joint Surg Br. 2002;84:141-144.

11. Benjamin M, Kaiser E, Milz S. Structure-function relationships in tendons: a review. J Anat. 2008;212:211-228.

12. Ackermann PW, Li J, Finn A, Ahmed M, Kreicbergs A. Autonomic innervation of tendons, ligaments and joint capsules. A morphologic and quantitative study in the rat. $J$ Orthop Res. 2001;19:372-378.

13. Bring DK, Kreicbergs A, Renstrom PA, Ackermann PW. Physical activity modulates nerve plasticity and stimulates repair after Achilles tendon rupture. J Orthop Res. 2007;25:164-172.

14. Shaw HM, Benjamin M. Structure-function relationships of enthuses in relation to mechanical load and exercise. Scand J Med Sci Sports. 2007; 17:303-315.

15. Zhang J, Wang JH. Characterization of differential properties of rabbit tendon stem cells and tenocytes. BMC Musculoskelet Disord. 2010;11:10.

16. Yao L, Bestwick CS, Bestwick LA, Maffulli N, Aspden RM. Phenotypic drift in human tenocyte culture. Tissue Eng. 2006;12(7):1843-1849.

17. Schwarz R, Colarusso L, Doty P. Maintenance of differentiation in primary cultures of avian tendon cells. Exp Cell Res.1976;102(1):63-71.

18. Jelinsky SA, Archambault J, Li L, Seeherman H. Tendon-selective genes identified from rat and human musculoskeletal tissues. J Orthop Res. 2010;28(3):289-297.

19. Wang A, Mackie K, Breidahl W, Wang T, Zheng MH. Evidence for the durability to autologous tenocyte injection for treatment of chronic resistant lateral epicondylitis: mean 4.5-year clinical follow-up. Am J Sports Med. 2015;43:1775-1783.

20. Clarke AW, Alyas F, Morris T, Robertson CJ, Bell J, Connell DA. Skinderived tenocyte-like cells for the treatment of patellar tendinopathy. Am J Sports Med. 2011;39(3):614-623.

21. Blum B, Benvensity N. The tumorigenicity of human embryonic stem cells. Adv Cancer Res. 2008;100:133-158.

22. Hentze H, Graichen R, Colman A. Cell therapy and the safety of embryonic stem cell-derived grafts. Trends Biotechnol. 2007;25:24-32.

23. Wyles SP, Yamada S, Oommen S, et al. Inhibition of DNA topoisomerase II selectively reduces the threat of tumorigenicity following induced pluripotent stem cell-based myocardial therapy. Stem Cell Dev. 2014;23(19):2274-2282.
24. Harding J, Mirochnitchenko O. Preclinical studies for induced pluripotent stem cell-based therapeutics. J Biol Chem. 2014;289(8):4585-4593.

25. Rony IK, Baten A, Bloomfield JA, Islam ME, Billah MM, Islam KD. Inducing pluripotency in vitro: recent advances and highlights in induced pluripotent stem cells generation and pluripotency reprogramming. Cell Prolif. 2015;48(2):140-156.

26. Guha P, Morgan JW, Mostoslavsky G, Rodrigues NP, Body AS. Lack of immune response to differentiated cells derived from syngeneic induced pluripotent stem cells. Cell Stem Cell. 2013;12(4):407-412.

27. Araki R, Uda M, Hoki Y, et al. Negligible immunogenicity of terminally differentiated cells derived from induced pluripotent or embryonic stem cells. Nature. 2013;494:100-104.

28. Zhao T, Zhang ZN, Rong Z, Xu Y. Immunogenicity of induced pluripotent stem cells. Nature. 2011;474(7350):212-215.

29. Taylor CJ, Bolton EM, Bradley JA. Immunological considerations for embryonic and induced pluripotent stem cell banking. Philos Trans $R$ Soc Lond B Biol Sci. 2011;366(1575):2312-2322.

30. Van Eijk F, Saris DB, Riesle J, et al. Tissue engineering for ligaments: a comparison of bone marrow stromal cells, anterior cruciate ligament, and skin fibroblasts as cell source. Tissue Eng. 2004;10:893-903.

31. Ge Z, Goh JC, Lee EH. Selection of cell source for ligament tissue engineering. Cell Transplant. 2005;14(8):573-583.

32. Hankemeier S, Hurschler C, Zeichen J, et al. Bone marrow stromal cells in a liquid fibrin matrix improve the healing process of patellar tendon window defects. Tissue Eng Part A. 2009;15(5):1019-1030.

33. Patrikoski M, Sivula J, Huhtala H, et al. Different culture conditions modulate the immunological properties of adipose stem cells. Stem Cells Transl Med. 2014;3(10):1220-1230.

34. Lui PP, Kong SK, Lau PM, et al. Immunogenicity and escape mechanisms of allogeneic tendon-derived stem cells. Tissue Eng Part A. 2014;20(21-22):3010-3020.

35. Lui PP, Kong SK, Lau PM, et al. Allogeneic tendon-derived stem cells promote tendon healing and suppress immunoreactions in hosts: in vivo model. Tissue Eng Part A. 2014;20(21-22):2998-3009.

36. Griffin MD, Ritter T, Mahon BP. Immunological aspects of allogeneic mesenchymal stem cell therapies. Hum Gene Ther. 2010; 21(12):1641-1655.

37. Manning CN, Martel C, Sakiyama-Elbert SE, et al. Adipose-derived mesenchymal stromal cells modulate tendon fibroblast responses to macrophage-induced inflammation in vitro. Stem Cell Res Ther. 2015;6:74.

38. Khubutiya MS, Vagabov AV, Temnov AA, Sklifas AN. Paracrine mechanisms of proliferative, anti-apoptotic and anti-inflammatory effects of mesenchymal stromal cells in models of acute organ injury. Cytotherapy. 2014;16(5):579-585.

39. Noack S, Seiffart V, Willbold E, Laggies S, Winkel A, ShahabOsterloh S. Periostin secreted by mesenchymal stem cells supports tendon formation in an ectopic mouse model. Stem Cells Dev. 2014; 23(16): 1844-1857.

40. Lange-Consiglio A, Rossi D, Tassan S, Perego R, Cremonesi F, Parolini O. Conditioned medium from horse amniotic membrane-derived multipotent progenitor cells: immunomodulatory activity in vitro and first clinical application in tendon and ligament injuries in vivo. Stem Cells Dev. 2013;22(22):3015-3024.

41. Sakaguchi Y, Sekiya I, Yagishita K, Muneta T. Comparison of human stem cells derived from various mesenchymal tissues: superiority of synovium as a cell source. Arthritis Rheum. 2005;52:2521-2529.

42. Harris MT, Butler DL, Boivin GP, Florer JB, Schantz EJ, Wenstrup RJ. Mesenchymal stem cells used for rabbit tendon repair can form ectopic bone and express alkaline phosphatase activity in constructs. J Orthop Res. 2004;22:998-1003.

43. Awad HA, Butler DL, Dressler MR, Smith F, Boivin GP, Young RG. Repair of patellar tendon injuries using mesenchymal stem cells and collagen scaffolds. J Orthop Res. 2003;21:420-431.

44. Tasso R, Augell A, Carida'M, et al. Development of sacromas in mice implanted with mesenchymal stem cells seeded onto bioscaffolds. Carcinogenesis. 2009;30(1):150-157. 
45. Guest DJ, Smith MR, Allen MR. Equine embryonic stem-like cells and mesenchymal stromal cells have different survival rates and migration patterns following their injection into damaged superficial digital flexor tendon. Equine Vet J. 2010;42(7):636-642.

46. Bruno S, Camussi G. Role of mesenchymal stem cell-derived microvesicles in tissue repair. Pediatr Nephrol. 2013;28(12):2249-2254.

47. Chen J, Li C, Chen L. The role of microvesicles derived from mesenchymal stem cells in lung diseases. Biomed Res Int. 2015;2015:985814.

48. Das S, Halushka MK. Extracellular vesicle microRNA transfer in cardiovascular disease. Cardiovasc Pathol. 2015;24(4):199-206.

49. Gutierrez Millan C, Colino Gandarillas CI, Sayalero Marinero ML, Lanao JM. Cell-based drug-delivery platforms. Ther Deliv. 2012; 3(1):25-41.

50. Gulotta LV, Kovacevic D, Ehteshami JR, Dagher E, Packer JD, Rodeo SA. Application of bone marrow-derived mesenchymal stem cells in a rotator cuff repair model. Am J Sports Med. 2009;37(11):2126-2133.

51. Valencia Mora M, Antuna Antuna S, Garcia Arranz M, Carrascal MT, Barco R. Application of adipose tissue-derived stem cells in a rat rotator cuff repair model. Injury. 2014;45 Suppl 4:S22-S27.

52. Barco R, Encinas C, Valencia M, Carrascal MT, Garcia-Arranz M, Antuna S. [Use of adipose-derived stem cells in an experimental rotator cuff fracture animal model]. Rev Esp Cir Ortop Traumatol. 2015; 59(1):3-8

53. Kida Y, Morihara T, Matsuda K, et al. Bone marrow-derived cells from the footprint infiltrate into the repaired rotator cuff. J Shoulder Elbow Surg. 2013;22(2):197-205.

54. Nourissat G, Diop A, Maurel N, et al. Mesenchymal stem cell therapy regenerates the native bone-tendon junction after surgical repair in a degenerative rat model. PLoS One. 2010;5(8):e12248.

55. Yokoya S, Mochizuki Y, Natsu K, Omae H, Nagata Y, Ochi M. Rotator cuff regeneration using a bioabsorbable material with bone marrowderived mesenchymal stem cells in a rabbit model. Am J Sports Med. 2012;40(6):1259-1268.

56. Oh JH, Chung SW, Kim SH, Chung JY, Kim JY. 2013 Neer Award: Effect of the adipose-derived stem cell for the improvement of fatty degeneration and rotator cuff healing in rabbit model. J Shoulder Elbow Surg. 2014;23(4):445-455.

57. Lee SY, Kim W, Lim C, Chung SG. Treatment of lateral epicondylosis by using allogeneic adipose-derived mesenchymal stem cells: a pilot study. Stem Cells. 2015;33:2995-3005.

58. Ilic N, Atkinson K. Manufacturing and use of human placenta-derived mesenchymal stromal cells for phase I clinical trials: establishment and evaluation of a protocol. Vojnosanit Pregl. 2014;71(7):651-659.

59. Hernigou P, Flouzat Lachaniette $\mathrm{CH}$, Delambre J, et al. Biologic augmentation of rotator cuff repair with mesenchymal stem cells during arthroscopy improves healing and prevents further tears: a casecontrolled study. Int Orthop. 2014;38(9):1811-1818.

60. Lee JY, Zhou Z, Taub PJ, et al. BMP-12 treatment of adult mesenchymal stem cells in vitro augments tendon-like tissue formation and defect repair in vivo. PLoS One. 2011;6(3):e17531.

61. Xu K, Al-Ani MK, Sun Y, et al. Platelet-rich plasma activates tendonderived stem cells to promote regeneration of Achilles tendon rupture in rats. J Tissue Eng Regen Med. Epub 2015 Mar 11.

62. Hoffmann A, Pelled G, Turgeman G, et al. Neotendon formation induced by manipulation of the Smad8 signalling pathway in mesenchymal stem cells. J Clin Invest. 2006;116(4):940-952.

63. Pelled G, Snedeker JG, Ben-Arav A, et al. Smad8/BMP2-engineered mesenchymal stem cells induce accelerated recovery of the biomechanical properties of the Achilles tendon. J Orthop Res. 2012; 30(12):1932-1939.

64. Tao X, Liu J, Chen L, Zhou Y, Tang K. EGR1 induces tenogenic differentiation of tendon stem cells and promotes rabbit rotator cuff repair. Cell Physiol Biochem. 2015;35(2):699-709.

65. Guerquin MJ, Charvet B, Nourissat G, et al. Transcription factor EGR1 directs tendon differentiation and promotes tendon repair. J Clin Invest. 2013;123(8):3564-3576.
66. Liu H, Zhang $\mathrm{C}$, Zhu S, et al. Mohawk promotes the tenogenesis of mesenchymal stem cells through activation of the TGF $\beta$ signaling pathway. Stem Cells. 2015;33(2):443-455.

67. Gulotta LV, Kovacevic D, Packer JD, Deng XH, Rodeo SA. Bone marrow-derived mesenchymal stem cells transduced with scleraxis improve rotator cuff healing in a rat model. Am J Sports Med. 2011; 39(6):1282-1289.

68. Gulotta LV, Kovacevic D, Montgomery S, Ehteshami JR, Packer JD, Rodeo SA. Stem cells genetically modified with the developmental gene MT1-MMP improve regeneration of the supraspinatus tendon-to-bone insertion site. Am J Sports Med. 2010;38(7):1429-1437.

69. Shearn JT, Juncosa-Melvin N, Boivin GP, et al. Mechanical stimulation of tendon tissue engineered constructs: effects on construct stiffness, repair biomechanics, and their correlation. J Biomech Eng. 2007; 129(6):848-854.

70. Juncosa-Melvin N, Shearn JT, Boivin GP, et al. Effects of mechanical stimulation on the biomechanics and histology of stem cell-collagen sponge constructs for rabbit patellar tendon repair. Tissue Eng. 2006; 12(8):2291-2300

71. Xu Y, Dong S, Zhou Q, et al. The effect of mechanical stimulation on the maturation of TDSCs-poly(L-lactide-co-e-caprolactone)/collagen scaffold constructs for tendon tissue engineering. Biomaterials. 2014; 35(9):2760-2772.

72. Zhang J, Li B, Wang JH. The role of engineered tendon matrix in the stemness of tendon stem cells in vitro and the promotion of tendon-like tissue formation in vivo. Biomaterials. 2011;32(29):6972-6981.

73. Zhang C, Yuan H, Liu H, et al. Well-aligned chitosan-based ultrafine fibers committed teno-lineage differentiation of human induced pluripotent stem cells for Achilles tendon regeneration. Biomaterials. 2015;53: 716-730.

74. Chen X, Yin Z, Chen JL, et al. Scleraxis-overexpressed human embryonic stem cell-derived mesenchymal stem cells for tendon tissue engineering with knitted silk-collagen scaffold. Tissue Eng Part A. 2014;20(11-12):1583-1592.

75. Zhang J, Wang JH. Platelet-rich plasma releasate promotes differentiation of tendon stem cells into active tenocytes. Am J Sports Med. 2010; 38(12):2477-2486.

76. Martinello T, Bronzini I, Perazzi A, et al. Effects of in vivo applications of peripheral blood-derived mesenchymal stromal cells (PB-MSCs) and platelet-rich plasma (PRP) on experimentally injured deep digital flexor tendons of sheep. J Orthop Res. 2013;31(2):306-314.

77. Chiou GJ, Crowe C, McGoldrick R, Hui K, Pham H, Chang J. Optimization of an injectable tendon hydrogel: the effects of plateletrich plasma and adipose-derived stem cells on tendon healing in vivo. Tissue Eng Part A. 2015;21(9-10):1579-1586.

78. Chen L, Dong SW, Liu JP, Tao X, Tang KL, Xu JZ. Synergy of tendon stem cells and platelet-rich plasma in tendon healing. J Orthop Res. 2012;30(6):991-997.

79. Chen L, Liu JP, Tang KL, et al. Tendon derived stem cells promote platelet-rich plasma healing in collagenase-induced rat Achilles tendinopathy. Cell Physiol Biochem. 2014;34(6):2153-2168.

80. Uysal CA, Tobita M, Hyakusoku H, Mizuno H. Adipose-derived stem cells enhance primary tendon repair: biomechanical and immunohistochemical evaluation. J Plast Reconstr Aesthet Surg. 2012; 65(12):1712-1719.

81. Gulotta LV, Kovacevic D, Packer JD, Ehteshami JR, Rodeo SA. Adenoviral-mediated gene transfer of human bone morphogenetic protein-13 does not improve rotator cuff healing in a rat model. Am J Sports Med. 2011;39(1):180-187.

82. Caliari SR, Harley BA. Structural and biochemical modification of a collagen scaffold to selectively enhance MSC tenogenic, chondrogenic, and osteogenic differentiation. Adv Healthc Mater. 2014;3(7): 1086-1096.

83. Hou Y, Mao Z, Wei X, et al. Effects of transforming growth factor-beta1 and vascular endothelial growth factor 165 gene transfer on Achilles tendon healing. Matrix Biol. 2009;28(6):324-335. 
84. Kraus TM, Imhoff FB, Wexel G, et al. Stem cells and basic fibroblast growth factor failed to improve tendon healing: an in vivo study using lentiviral gene transfer in a rat model. J Bone Joint Surg Am. 2014;96(9):761-769.

85. Sole A, Spriet M, Padgett KA, et al. Distribution and persistence of technetium-99 hexamethyl propylene amine oxime-labelled bone marrow-derived mesenchymal stem cells in experimentally induced tendon lesions after intratendinous injection and regional perfusion of the equine distal limb. Equine Vet J. 2013;45(6):726-731.

86. Becerra P, Valdes Vazquez MA, Dudhia J, et al. Distribution of injected technetium(99 m)-labeled mesenchymal stem cells in horses with naturally occurring tendinopathy. J Orthop Res. 2013;31(7):1096-1102.

87. Awad HA, Butler DL, Harris MT, et al. In vitro characterization of mesenchymal stem cell-seeded collagen scaffolds for tendon repair: effects of initial seeding density on contraction kinetics. J Biomed Mater Res. 2000;51(2):233-240.

88. Scharf A, Holmes S, Thoresen M, Mumaw J, Stumpf A, Peroni J. Superparamagnetic iron oxide nanoparticles as a means to track mesenchymal stem cells in a large animal model of tendon injury. Contrast Media Mol Imaging. 2015;10:388-397.

89. Liu H, Zhang C, Zhu S, et al. Mohawk promotes the tenogenesis of mesenchymal stem cells through activation of the TGF $\beta$ signaling pathway. Stem Cells. 2015;33(2):443-455.

90. Colosimo A, Curini V, Russo V, et al. Characterization, GFP gene nucleofection, and allotransplantation in injured tendons of ovine amniotic fluid-derived stem cells. Cell Transplant. 2013;22(1):99-117.

91. Chen HS, Su YT, Chan TM, et al. Human adipose-derived stem cells accelerate the restoration of tensile strength of tendon and alleviate the progression of rotator cuff injury in a rat model. Cell Transplant. 2015;24(3):509-520.
92. Brown JP, Galassi TV, Stoppato M, Schiele NR, Kuo CK. Comparative analysis of mesenchymal stem cell and embryonic tendon biochemical and mechanical factors. Stem Cell Res Ther. 2015;6:89.

93. Tang QM, Chen JL, Shen WL, et al. Fetal and adult fibroblasts display intrinsic differences in tendon tissue engineering and regeneration. Sci Rep. 2014;4:5515.

94. Liu H, Zhu S, Zhang C, et al. Crucial transcription factors in tendon development and differentiation: their potential for tendon regeneration. Cell Tissue Res. 2014;356(2):287-298.

95. Lee CH, Lee FY, Tarafder S, et al. Harnessing endogenous stem/progenitor cells for tendon regeneration. J Clin Invest. 2015;125(7):2690-2701.

96. Lui PP. Identity of tendon stem cells - how much do we know? Identity of tendon stem cells - how much do we know? J Cell Mol Med. 2013;17(1):55-64.

97. Durant TJ, Dyment N, McCarthy MB, et al. Mesenchymal stem cell response to growth factor treatment and low oxygen tension in 3-dimensional construct environment. Muscles Ligaments Tendons J. 2014;4(1):46-51.

98. Lee WY, Lui PP, Rui YF. Hypoxia-mediated efficient expansion of human tendon-derived stem cells in vitro. Tissue Eng Part A. 2012; 18(5-6):484-498.

99. Glenn JD, Whartenby KA. Mesenchymal stem cells: emerging mechanisms of immunomodulation and therapy. World J Stem Cells. 2014; 6(5):526-539.
Stem Cells and Cloning: Advances and Applications

\section{Publish your work in this journal}

Stem Cells and Cloning: Advances and Applications is an international, peer-reviewed, open access journal. Areas of interest in stem cell research include: Embryonic stem cells; Adult stem cells; Blastocysts; Cordblood stem cells; Stem cell transformation and culture; Therapeutic cloning; Umbilical cord blood and bone marrow cells; Laboratory,

\section{Dovepress}

animal and human therapeutic studies; Philosophical and ethical issues related to stem cell research. This journal is indexed on CAS. The manuscript management system is completely online and includes a quick and fair peer-review system. Visit http://www.dovepress.com/ testimonials.php to read real quotes from published authors. 